\title{
Non-Clinical Postnatal Trial Phase
}

National Cancer Institute

\section{Source}

National Cancer Institute. Non-Clinical Postnatal Trial Phase. NCI Thesaurus. Code C124604.

The protocol defined period of time in a study that begins immediately after the birth of the subject. 